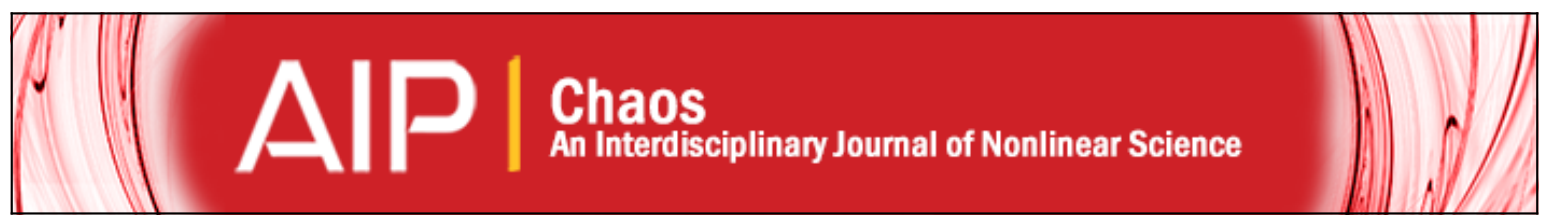

\title{
Dynamical properties of a dissipative hybrid Fermi-Ulam-bouncer model
}

Denis G. Ladeira and Edson D. Leonel

Citation: Chaos: An Interdisciplinary Journal of Nonlinear Science 17, 013119 (2007); doi: 10.1063/1.2712014 View online: http://dx.doi.org/10.1063/1.2712014

View Table of Contents: http://scitation.aip.org/content/aip/journal/chaos/17/1?ver=pdfcov

Published by the AIP Publishing

Alp Re-register for Table of Content Alerts

\section{Create a profile.}




\title{
Dynamical properties of a dissipative hybrid Fermi-Ulam-bouncer model
}

\author{
Denis G. Ladeira \\ Departamento de Física, ICEx, Universidade Federal de Minas Gerais, UFMG, CP 702, \\ 30.123-970, Belo Horizonte, MG Brazil \\ Edson D. Leonel \\ Departamento de Estatística, Matemática Aplicada e Computação, IGCE, Universidade Estadual Paulista, \\ UNESP, Av. 24A, 1515, Bela Vista 13.506-700, Rio Claro, SP Brazil
}

(Received 5 October 2006; accepted 5 February 2007; published online 27 March 2007; publisher error corrected 30 March 2007)

\begin{abstract}
Some consequences of dissipation are studied for a classical particle suffering inelastic collisions in the hybrid Fermi-Ulam bouncer model. The dynamics of the model is described by a twodimensional nonlinear area-contracting map. In the limit of weak and moderate dissipation we report the occurrence of crisis and in the limit of high dissipation the model presents doubling bifurcation cascades. Moreover, we show a phenomena of annihilation by pairs of fixed points as the dissipation varies. (C) 2007 American Institute of Physics. [DOI: 10.1063/1.2712014]
\end{abstract}

We considered the problem of a classical particle confined between two rigid walls and suffering inelastic collisions with them in the presence of a constant gravitational field. One of the walls is fixed and the position of the other one varies periodically in time. This model was recently introduced in the literature [Leonel and McClintock, J. Phys. A 38, 823 (2005)] and it is called the hybrid Fermi-Ulam-bouncer model. It shows features of both Fermi-Ulam and bouncer models for distinct regions of the phase space and consider different ranges of the external gravitational field. The inelastic collisions carry the dynamics to show the property of area contraction with the possibility of observing chaotic attractors and sinks. Specifically, we characterize events of the crisis as the damping coefficients are varied. Moreover, in the limit of high dissipation the system exhibits a sequence of doubling bifurcation cascade. We also report the annihilation of pairs of fixed points as the damping coefficients are varied.

\section{INTRODUCTION}

The investigation of nonlinear systems has received special interest in the last years mainly because of the prediction and/or explanation of the often unexpected phenomenon that they can exhibit. Besides, significant advances towards the understanding of the behavior of such systems ${ }^{1,2}$ generally lead to a better description of both their qualitative and quantitative asymptotic behaviors. Included in these sort of systems are the conservative time-dependent Hamiltonian systems and also the conservative billiard problems. Generally they present a mixed phase space structure with the presence of invariant spanning curves, and chaotic seas which are generally surrounding Kolmogorov-Arnold-Moser (KAM) islands. Such phase space is indeed a property of nondegenerate Hamiltonian systems. However the introduction of dissipation in such models yields the dynamics to be very different from that of the nondissipative case. It is especially possible to observe attractors including sinks and chaotic at- tractors. They can be observed given that in most cases, the property of area preservation, as observed in nondissipative problems, is no longer matched.

In this paper we revisit the classical hybrid Fermi-Ulam bouncer model ${ }^{3}$ seeking to understand and describe some of its dynamical properties under the effect of inelastic collisions. The model consists of a classical particle which is confined to bounce between two rigid walls. One is assumed to be fixed while the other one moves periodically in time. Moreover, there is the presence of a constant gravitational field. We emphasize that, without loss of generality, the external field could be, in principle, a constant electric field that acts on a charged particle. In the limit of high dissipation the model presents doubling period bifurcations as the values of the dimensionless external field or amplitude of oscillation is changed. By varying the damping parameters there are also observed crisis events, which are characterized by crossing of stable and unstable manifolds and destruction of the chaotic attractor. The study of such crisis events are of main interest in many real experimental systems and in studies in which it is desired the control over the chaos. Locking (phenomena in which the particle and the oscillating wall move together) is also observed for some values of $g$ and $\epsilon$ in the limit of high dissipation and, moreover, pairs of fixed points self-destroy as the dissipation rises. The studies on the onedimensional Fermi accelerator model began in 1949 (Ref. 4) when Enrico Fermi attempted to describe a mechanism in which cosmic rays could acquire their enormous energy. After that, his original model (also called the Fermi-Ulam model, a classical particle bouncing between two rigid walls in the absence of any external fields; one wall is fixed and the other one is time dependent) was modified to account for example, a simplified version, ${ }^{5}$ to include the presence of an external constant gravitational field, ${ }^{6}$ inelastic collisions, ${ }^{7-12}$ and even frictional force ${ }^{13,14}$ (see also Refs. 15 and 16 for recent results on the nondissipative Fermi-Ulam model). For the case of inelastic collisions, it was shown that the property 
of area preservation is broken and it is possible to observe in the system the presence of chaotic attractors (sometimes also called strange attractors) and sinks. In Ref. 7 Everson showed the occurrence of doubling cascade bifurcation for the bouncer model. The bouncer model is a slightly modified version of the Fermi-Ulam in which the returning mechanism for the next collision with the moving wall is due only to the gravitational field. Similar results were observed in the presence of a frictional force when Luna-Acosta ${ }^{13}$ analytically accounted for a dimensional reduction on the twodimensional mapping. He too observed and described with many details the attainment of the doubling bifurcation cascade. In their results, however, and as an attempt to avoid having multiple collisions with the same wall, they have described the oscillating wall by an expression that does not allow the wall to change the signal of its velocity. Thus, depending on the experimental apparatus, their results can be difficult to be obtained in a laboratory for long times, as it is often necessary in practice. Our approach to the problem of the dissipative hybrid Fermi-Ulam-bouncer model considers the dynamics of the complete version and thus no modifications or simplifications were introduced. The main applicability of the complete version lies on the high dissipation regime, in which the locking phenomena can take place and, therefore, the successive collisions are important and cannot be neglected.

The paper is organized as follows: In Sec. II, we describe all the steps used in the mapping derivation. The numerical results are presented and discussed in Sec. III while Sec. IV summarizes our results and presents concluding remarks.

\section{THE MODEL AND MAPPING DERIVATION}

We now describe some details of the model and the procedure used to obtain the nonlinear mapping. The system consists of a classical particle confined between and bouncing inelastically with two rigid walls. One of which is assumed to be fixed at position $y=l$ and the other one is moving in time according to $y_{w}=\varepsilon \cos (\omega t)$ where $\varepsilon$ and $\omega$ are the corresponding amplitude and frequency of oscillation, respectively. Additionally, there is an external constant gravitational field $g^{\prime}$. It is assumed that the ball collides inelastically with the two walls. The restitution coefficient of the upper wall is $\alpha$ while for the moving wall is $\beta$. The case of $\alpha=\beta=1$ recovers all the results of the nondissipative case. Therefore, the completely inelastic collisions are accounted for when $\alpha=0$ or $\beta=0$ and have different implications. In the case of $\alpha=0$ immediately after the collision with the upper wall the ball goes in the downward direction with null reflected velocity. Besides, depending on the values of the control parameters, for the case $\beta=0$ the particle can, for example, move together with the time varying wall or be relaunched with the maximum moving wall velocity. We are interested in the limits of both $\alpha$ and $\beta$ in the range $[0,1)$. On the case of completely inelastic collisions, it is possible to observe the phenomenon of locking, as can be seen in Refs. 10 and 11. Before discussing the procedures of the mapping derivation it is worth stressing that the dynamical variables of the mapping are $\left(v_{n}, t_{n}\right)$, where $v_{n}$ is the velocity of the particle in the $n$th collision with the moving wall at the instant $t_{n}$, thus the mapping is given as $T\left(v_{n}, t_{n}\right)$ $=\left(v_{n+1}, t_{n+1}\right)$. Moreover there are three different possible situations allowed in the ball: (i) the particle experiences multiple hits with the moving wall before leaving the collision zone, which is given by the region in the interval $[-\varepsilon, \varepsilon]$; (ii) the particle leaves the collision zone but does not have enough energy to reach the upper wall and returns in the downward direction due only to the gravitational field; (iii) the particle has enough energy and hits the upper wall and, after suffering a partial loss of energy, returns downward due to both the action of the reflection on the fixed wall as well as due to the gravitational field. It is also interesting to consider dimensionless variables and then we define $\epsilon=\varepsilon / l, g=g^{\prime} /\left(\omega^{2} l\right), V_{n}=v_{n} /(\omega l)$ and measure the time in terms of the number of oscillations of the moving wall $\phi_{n}=\omega t$. With these definitions, the mapping is written as

$$
T:\left\{\begin{array}{l}
V_{n+1}=\beta\left(V_{n}^{*}+g \phi_{c}\right)-(1+\beta) \epsilon \sin \left(\phi_{n+1}\right), \\
\phi_{n+1}=\phi_{n}+\Delta T_{n} \bmod 2 \pi .
\end{array}\right.
$$

where the expressions of $V_{n}^{*}$ and $\Delta T_{n}$ depend on what kind of collision occurs. For case (i) the expressions are $V_{n}^{*}=-V_{n}$ and $\Delta T_{n}=\phi_{c}$, where $\phi_{c}$ is obtained by requesting that the position of the moving wall be equal to the particle's position. Such a condition gives rise to the following transcendental equation that must be numerically solved:

$$
G\left(\phi_{c}\right)=\epsilon \cos \left(\phi_{n}+\phi_{c}\right)-\epsilon \cos \left(\phi_{n}\right)-V_{n} \phi_{c}+\frac{g \phi_{c}^{2}}{2} .
$$

For case (ii), which occurs when the particle leaves the collision zone with $V_{n} \leq \sqrt{-2 g z_{n}}$, where $z_{n}=\epsilon \cos \left(\phi_{n}\right)-1$, the expressions are $V_{n}^{*}=\sqrt{V_{n}^{2}+2 g \epsilon\left[\cos \left(\phi_{n}\right)-1\right]}$ and $\Delta T_{n}=\phi_{u}$ $+\phi_{d}+\phi_{c}$ where $\phi_{u}=V_{n} / g$ denotes the time that the particle spends traveling in the upward direction. On the other hand, $\phi_{d}=\sqrt{2 \epsilon\left[\cos \left(\phi_{n}\right)-1\right] / g+V_{n}^{2} / g^{2}}$ represents the elapsed time in the downward direction up to the entrance of the collision zone. The term $\phi_{c}$ is numerically obtained from the equation

$$
F\left(\phi_{c}\right)=\epsilon \cos \left(\phi_{n}+\phi_{u}+\phi_{d}+\phi_{c}\right)-\epsilon+V_{n}^{*} \phi_{c}+\frac{g \phi_{c}^{2}}{2},
$$

which corresponds to the condition of the particle's position being the same as the moving wall position. Finally, case (iii) is accounted for the condition $V_{n}>\sqrt{-2 g z_{n}}$. The auxiliary expressions are $V_{n}^{*}=\sqrt{\alpha^{2}\left(V_{n}^{2}+2 g z_{n}\right)+2 g(1-\epsilon)}$ and $\Delta T_{n}=\phi_{u}+\phi_{d}+\phi_{c} \quad$ with $\quad \phi_{u}=V_{n} / g-\sqrt{V_{n}^{2} / g^{2}+2 z_{n} / g} \quad$ and $\phi_{d}=\sqrt{\alpha^{2}\left(V_{n}^{2} / g^{2}+2 z_{n} / g\right)+2(1-\epsilon) / g}-\alpha \sqrt{V_{n}^{2} / g^{2}+2 z_{n} / g}$. The value of $\phi_{c}$ is obtained from the same Eq. (3) using, however, the expressions of $\phi_{u}, \phi_{d}$, and $V_{n}^{*}$ given by condition (iii).

Evaluating the determinant of the Jacobian matrix $J$, we found that both cases (i) and (ii) show that

$$
\operatorname{det} J=\beta^{2}\left[\frac{V_{n}+\epsilon \sin \left(\phi_{n}\right)}{V_{n+1}+\epsilon \sin \left(\phi_{n+1}\right)}\right],
$$

and case (iii) has 


$$
\operatorname{det} J=\beta^{2} \alpha^{2}\left[\frac{V_{n}+\epsilon \sin \left(\phi_{n}\right)}{V_{n+1}+\epsilon \sin \left(\phi_{n+1}\right)}\right] .
$$

The case of measure preservation is recovered only for the condition $\alpha=\beta=1$.

\section{NUMERICAL RESULTS}

Let us now discuss some of the numerical results obtained for the dissipative version of the hybrid Fermi-Ulam bouncer model. One of the most drastic consequences of the introduction of dissipation via inelastic collisions is the destruction of the mixed phase space structure. Besides, we shall discuss the results obtained in two limits of dissipation: (a) weak up to moderate dissipation and (b) high dissipation. In the regime (a) we illustrate some consequences of the dissipation by investigating and characterizing events of the boundary crisis.

\section{A. Weak and moderate dissipation}

One of the signatures of a boundary crisis ${ }^{17,18}$ is a sudden destruction of the chaotic attractor given by a crossing of stable and unstable manifolds generated from the same saddle point. Moreover and since the phase space now contracts area, it is possible to observe attractors in the dynamics. They can be just sinks (attracting fixed points) or even chaotic attractors. However, to classify such fixed points, we must first know their corresponding expressions. Thus, the conditions of $V_{n+i}=V_{n}$ and $\phi_{n+i}=\phi_{n}$ give a fixed point if $i$ $=1$, period two orbit if $i=2$, and so on. From the mapping defined in the previous section it is possible to obtain the fixed points for both situations (ii) and (iii). However, in the limit of weak and moderate dissipation, attracting fixed points are observed only for the situation (iii) while the attracting fixed points related to case (ii) are observed only in the limit of high dissipation, which will be described in Sec. III B. Thus, evaluating the fixed points for situation (iii) we obtain that the expression of the velocity is given by

$$
\begin{aligned}
V= & \frac{1+\beta}{\gamma} \epsilon \sin (\phi) \\
& -\frac{\beta}{\gamma} \sqrt{\alpha^{2}(1+\beta)^{2} \epsilon^{2} \sin ^{2}(\phi)+2 \gamma g\left(1-\alpha^{2}\right) z},
\end{aligned}
$$

where $\gamma=\beta^{2} \alpha^{2}-1$ and $z=\epsilon \cos (\phi)-1$. The expression of $\phi$, however, can be obtained only after the numerical solution of $h(\phi)=0$, where $h(\phi)$ is given by

$$
\begin{aligned}
h(\phi)= & V-(1+\alpha) \sqrt{V^{2}+2 g z}-2 \pi m g \\
& +\sqrt{\alpha^{2}\left(V^{2}+2 g z\right)-2 g z},
\end{aligned}
$$

with $m=1,2,3, \ldots$.

The knowledge of the saddle point (even the numerical value) allow us to obtain its corresponding invariant manifolds. Thus, shown in Fig. 1(a) is the behavior of the manifolds generated from a saddle point for $m=1$ in Eqs. (6) and (7). It is easy to see in Fig. 1(a) that there are four branches of the invariant manifolds, which were obtained by iteration of a set of different initial conditions near the saddle point and distributed along the eigenvectors of the saddle point.
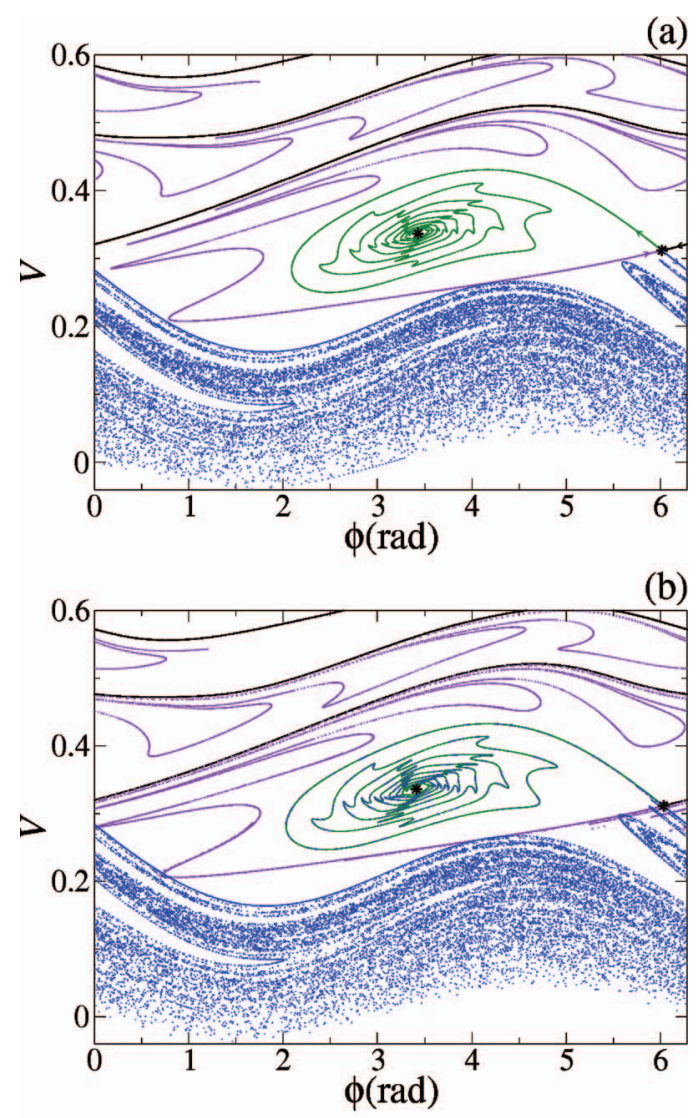

FIG. 1. (Color) Stable and unstable manifolds for the saddle point for $m$ $=1, \quad \epsilon=0.04, \quad g=10^{-4}$ and (a) $\alpha=0.96625, \quad \beta=0.96625$ and (b) $\alpha$ $=0.968125, \beta=0.968125$.

Such eigenvectors give the initial direction of the manifolds. The unstable manifolds are generated by trajectories heading away from the fixed point and are obtained by the direct iteration of mapping $T$ given by Eq. (1). As it is shown in Fig. 1(a), the evolution of the upward branch of the unstable manifold goes into an attracting sink while the downward direction moves asymptotically into a chaotic attractor. On the other hand, the stable manifolds correspond to trajectories heading directly toward the saddle point. The construction of the stable manifolds are not so straightforward as for the unstable manifolds since they depend on the inverse of the mapping, $T^{-1}$, i.e., $T^{-1}\left(V_{n+1}, \phi_{n+1}\right)=\left(V_{n}, \phi_{n}\right)$. We are not reporting the expressions of the inverse mapping in this paper but we stress that they request a detailed numerical simulation. Despite the slight modification of the damping coefficients, Fig. 1(b) illustrates the crossing of the stable and unstable manifolds of the saddle point for $m=1$. Such crossing gives birth to a homoclinic orbit and consequently the chaotic attractor is destroyed via a boundary crisis. The event of the boundary crisis, however, is not limited to $m=1$ and it is possible to characterize this kind of crisis for many other values of $m$. As an illustration and to reinforce the generality of the procedure, shown in Fig. 2 is the occurrence of a boundary crisis for $m=2$. We note in Fig. 2(a) that there are in principle three attractors: two of them are sinks corresponding to $m=1$ and $m=2$ and a chaotic attractor. Moreover, the stable manifolds define the boundaries for the basin 


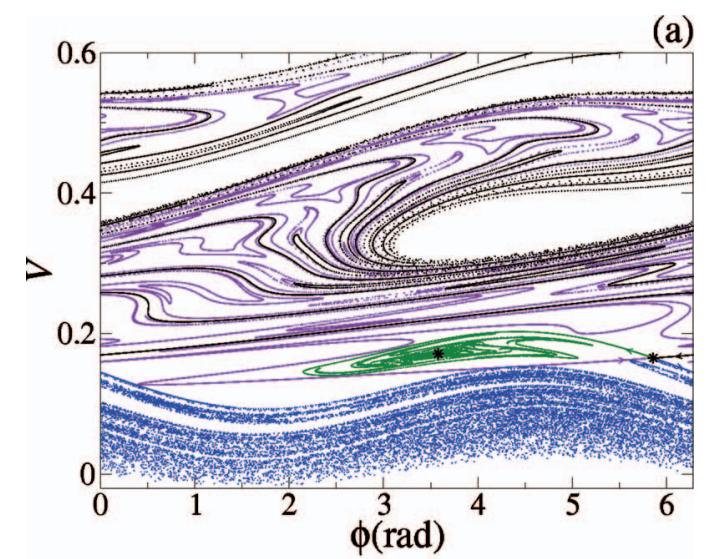

(b)

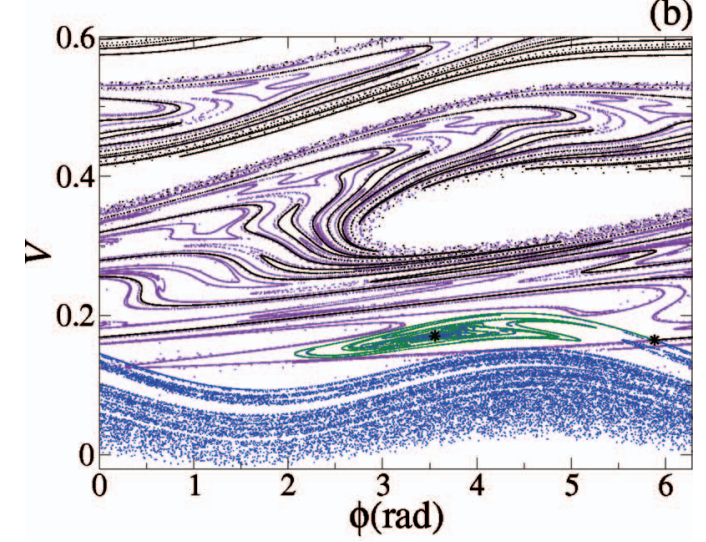

FIG. 2. (Color) Corresponding stable and unstable manifolds for the saddle point for $m=2, \epsilon=0.02, g=10^{-4}$ and (a) $\alpha=0.9, \beta=1$ and (b) $\alpha=0.905$, $\beta=1$.

of attraction of each attractor. A boundary crisis in Fig. 2(b) is evident with the stable manifold of the saddle for $m=2$ crossing the corresponding unstable manifold emanating from the same saddle point. The corresponding basin of attraction for the three attractors shown in Fig. 2(a) are illustrated in Fig. 3. On the construction of Fig. 3, we have used a grid of 200 different initial conditions on the range $V_{0}$ $\in[0,0.6]$ and the other 200 for $\phi_{0} \in[0,2 \pi)$. Thus in total

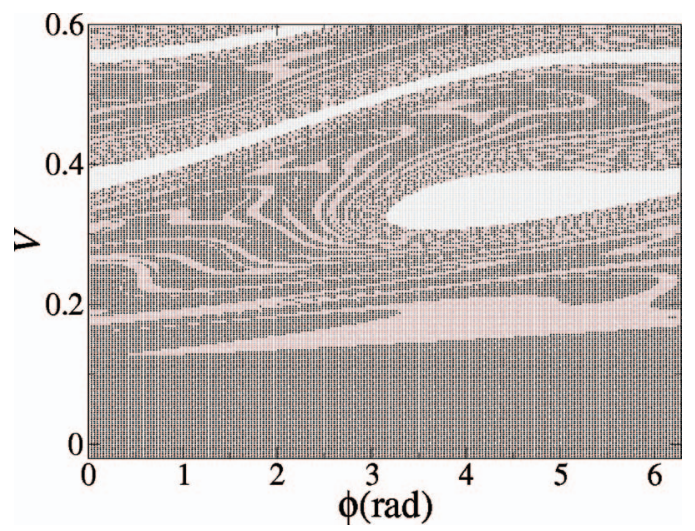

FIG. 3. (Color) Basin of attraction for the three attractors shown in Fig. 2(a). The basin of attraction of the chaotic attractor is shown in black, while the basin of attraction of the attracting fixed point corresponding to $m=1$ is shown in gray and for $m=2$ in brown. The control parameters used were the same as those of Fig. 2(a).
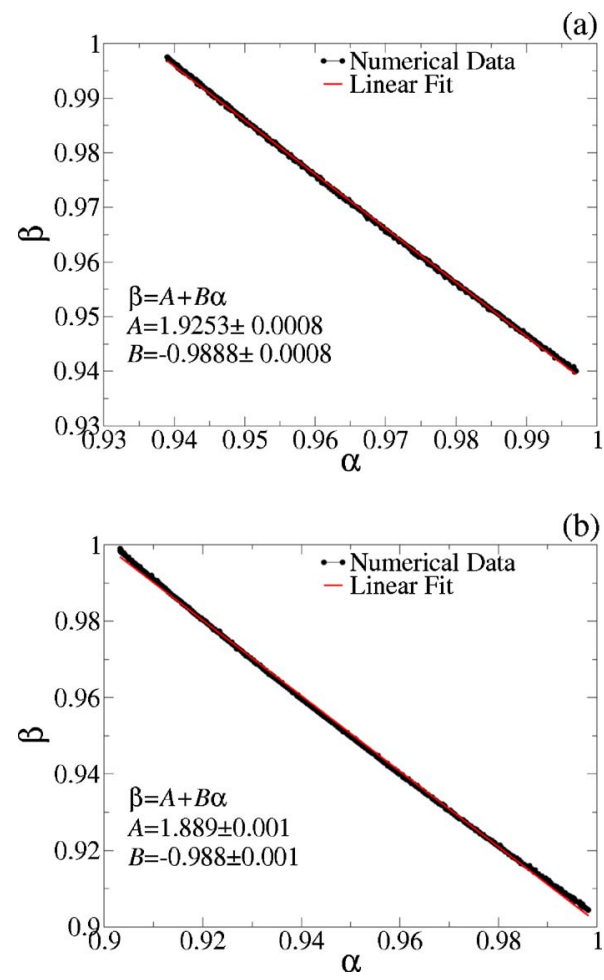

FIG. 4. Family of crisis for (a) $m=1$ and (b) $m=2$ in the dissipative hybrid Fermi-Ulam bouncer model. The control parameters used were (a) $\epsilon=4$ $\times 10^{-2}$ and $g=10^{-4}$; (b) $\epsilon=2 \times 10^{-2}$ and $g=10^{-4}$.

we have iterated $4 \times 10^{4}$ orbits over $10^{5}$ collisions with the moving wall. One might observe that considering the range of initial conditions as described above, all initial conditions will be attracted to the attracting fixed point for $m=1$ and $m=2$ as well as to the chaotic attractor. It is important to emphasize that other periodic orbits could in principle also exist for those combinations of control parameters used in Fig. 3. We stress however if such periodic orbits exist, their basin of attraction must be very small.

Since we have considered two damping coefficients in the model, $\alpha$ and $\beta$, it is possible to combine both in order to have an effective damping coefficient. This combination can be important and shows great applicability in experiments where dissipation cannot be neglected in practice. If the hypothesis of an effective damping coefficient is correct, the boundary crisis, as characterized in Figs. 1(a) and 1(b) could be obtained for other combinations of $\alpha$ and $\beta$, as for example, raising the value of $\alpha$ (which is equivalent to reducing the dissipation) and reducing $\beta$ (which corresponds to increasing the dissipation). This is indeed the case, as can be seen in Fig. 4(a). Such a result allows us to describe a family of boundary crisis according to the relation $\beta=A+B \alpha$. A linear fit gives $A=1.9253 \pm 0.0008$ and $B=-0.9888 \pm 0.0008$. Similar results can also be observed for a boundary crisis corresponding to $m=2$, as shown in Fig. 4(b). The linear fit furnishes $A=1.889 \pm 0.001$ and $B=-0.988 \pm 0.001$. We have considered in the construction of Figs. 4(a) and 4(b), a grid of $500 \times 500$ different values for the control parameters $\alpha$ and $\beta$, as labeled in the figure. The initial condition, chosen along the downward branch of the unstable manifold, was iterated up to $10^{6}$ collisions with the moving wall. The nu- 
merical criteria used to establish the crisis event consists of checking whether the particle makes an incursion toward the attracting sink and then approaches it being captured. The interested reader that wants to address the question of the transient time immediately after the boundary crisis might look at Ref. 19.

\section{B. Results for the limit of high dissipation}

Let us now discuss our results in the limit of high dissipation. We consider as a high dissipation the situation in which the particle, in the collision process, loses more than half of its velocity. As an example, if $\beta=0.4$, then the velocity of the particle immediately after the impact is, in the reference frame of the moving wall, $60 \%$ smaller than the value immediately before the collision. In some of the previous results in the literature, as addressed by Everson ${ }^{7}$ and Luna-Acosta, ${ }^{13}$ they have observed the phenomenon of doubling bifurcation cascade for high dissipation limit. The Everson's results were obtained for inelastic collisions while Luna-Acosta's takes into account the presence of frictional force. Both consider the bouncer model. Their results confirm that, despite the dissipative force being of a different kind (in the inelastic collisions the dissipative force acts only at the instant of the collision contrary to the frictional force that acts along all the particle's trajectory) in the high limit of the damping coefficient for both dissipative versions, the doubling cascade in the variable $\phi$ was obtained. However, they have imposed that the moving wall velocity assume an expression basically of the type $V_{w}=c(1+\sin (t))$, where $c$ is a constant. Such an expression allows them to avoid problems with numerical implementation of the multiple collisions that might arise on the complete version. Depending on the value of the external gravitational field (weak fields for example) and on the strength of the damping coefficient, the multiple collisions can be rare, thus their approximation is completely justified for the limit of high dissipation. In the hybrid Fermi-Ulam bouncer model, however, the successive collisions cannot be neglected in the limit of high dissipation since the particle might in principle spend much more time in the low energy regime, thus suffering many more multiple collisions when compared to the nondissipative case.

We present in Fig. 5 some results for the limit of high dissipation when the external field $g$ varies while the other parameters are fixed at the values $\epsilon=4 \times 10^{-2}, \beta=0.4$, and $\alpha=0.99$. In Fig. 5(a) we present the doubling cascade for the variable phase, $\phi$, and in Fig. 5(b) is shown the period doubling sequence for the velocity of the particle.

We have also obtained the Lyapunov exponent, $\lambda$, associated with these period doubling sequences, as presented in Fig. 5(c). It is well known that the Lyapunov exponent shows great applicability as a practical tool that can quantify the average expansion or contraction rate for a small volume of initial conditions. As discussed in Ref. 20, the Lyapunov exponents are defined as
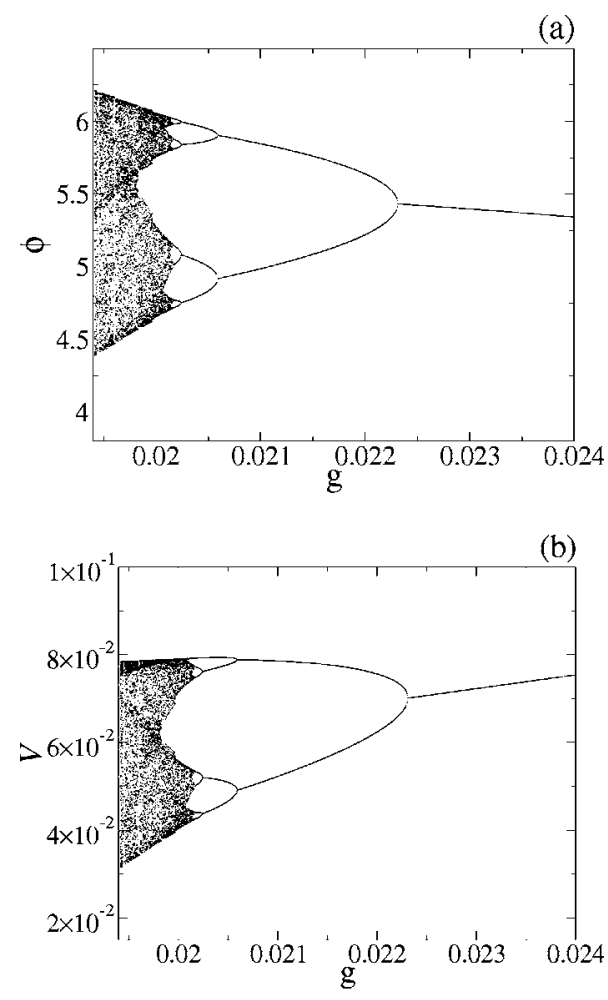

(c)

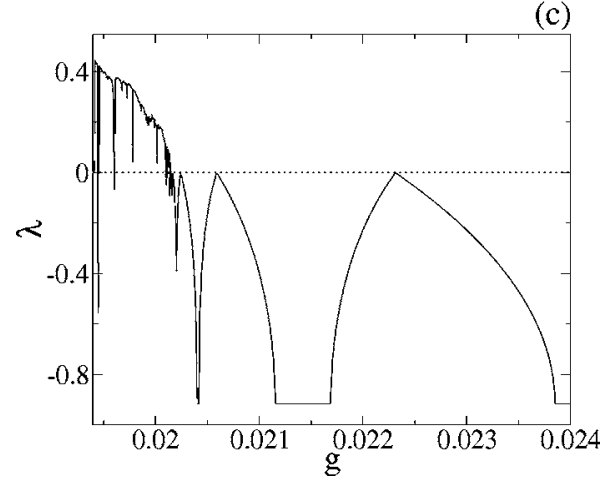

FIG. 5. Doubling period sequence of (a) the phase and (b) the velocity of the particle when $g$ is the variable parameter. (c) The Lyapunov exponent associated with the doubling cascades shown in (a) and (b).

$$
\lambda_{j}=\lim _{n \rightarrow \infty} \frac{1}{n} \ln \left|\Lambda_{j}\right|, \quad j=1,2,
$$

where $\Lambda_{j}$ are the eigenvalues of $M=\prod_{i=1}^{n} J_{i}\left(V_{i}, \phi_{i}\right)$ and $J_{i}$ is the Jacobian matrix evaluated over the orbit $\left(V_{i}, \phi_{i}\right)$. It is important to stress that a direct implementation of a computational algorithm to evaluate Eq. (8) has a severe limitation to obtain $M$. Even in the limit of short $n$, the components of $M$ can assume very different orders of magnitude for chaotic orbits and periodic attractors, yielding the impractical implementation of the algorithm. In order to avoid such problems, we note that $J$ can be written as $J=\Theta T$ where $\Theta$ is an orthogonal matrix and $T$ is a right triangular matrix. Thus we rewrite $M$ as $M=J_{n} J_{n-1}, \ldots, J_{2} \Theta_{1} \Theta_{1}^{-1} J_{1}$, where $T_{1}=\Theta_{1}^{-1} J_{1}$. A product of $J_{2} \Theta_{1}$ defines a new $J_{2}^{\prime}$. In a next step, it is easy to show that $M=J_{n} J_{n-1}, \ldots, J_{3} \Theta_{2} \Theta_{2}^{-1} J_{2}^{\prime} T_{1}$. The same procedure can be used to obtain $T_{2}=\Theta_{2}^{-1} J_{2}^{\prime}$, and so on. Using this procedure, the problem is reduced to evaluate the diagonal ele- 
ments of $T_{i}: T_{11}^{i}, T_{22}^{i}$. Finally, the Lyapunov exponents are now given by

$$
\lambda_{j}=\lim _{n \rightarrow \infty} \frac{1}{n} \sum_{i=1}^{n} \ln \left|T_{j j}^{i}\right|, \quad j=1,2 .
$$

If at least one of the $\lambda_{j}$ is positive, then the orbit is classified as chaotic. Therefore we define $\lambda$ as the larger value of the $\lambda_{j}$. As we can observe in Fig. 5, the periodic attractors are characterized by negative values of the Lyapunov exponent. It is also observed that $\lambda$ goes to zero at the values of $g$ for which the bifurcations occur. Moreover, the Lyapunov exponent assumes positive values beyond a critical value of the parameter $g$ characterizing, thus, the values of the external field for which chaotic orbits are observed. The initial conditions used in the construction of Figs. 5(a)-5(c) were $\phi_{0}$ $=5.5$ and $V_{0}=0.07$. As a technical detail, in the construction of Figs. 5(a) and 5(b), we have disregarded the first $10^{5}$ collisions with the moving wall as an attempt to eliminate the transient behavior. Another interesting observation is that the Lyapunov exponent assumes a constant and negative value for extents and distinct ranges of the control parameter $g$. The explanation for such behavior, as it was shown previously by Everson, ${ }^{7}$ is that the eigenvalues of the Jacobian matrix become complex numbers. The bifurcation cascades are observed for $g \in[0.019,0.0287]$. For values of $g$ out of such an interval and in the limit of high dissipation the particle locks its motion with the motion of the oscillating wall.

The period-1 window observed in Figs. 5(a) and 5(b) corresponds to the fixed point of the situation (ii) in which the particle leaves the collision zone but does not have enough energy to reach the upper wall and falls downward due to the action of the gravitational field. This fixed point can be evaluated by requiring the condition $V_{n+1}=V_{n}$ and $\phi_{n+1}=\phi_{n}$ in Eq. (1) employing, however, the expressions of $V_{n}^{*}$ and $\Delta T_{n}$ obtained for the situation (ii) described in Sec. II. The expressions of such fixed point are given by

$$
\phi=\arcsin \left[\frac{V}{\epsilon}\left(\frac{\beta-1}{\beta+1}\right)\right],
$$

$$
V=\pi m g
$$

for $m=1,2,3, \ldots$. The period-1 window in Figs. 5(a) and 5(b) corresponds to the fixed point for $m=1$. Since the argument of the arcsin function in Eq. (10) cannot acquire a value larger than the unity, for the combination of control parameters used in Fig. 5, we cannot have fixed points for $m>1$.

Similarly, the doubling bifurcation cascades can also be obtained as a function of the control parameter $\epsilon$ for fixed values of $g, \alpha$, and $\beta$ as they are shown in Fig. 6. The initial conditions used in the construction of Fig. 6 were $V_{0}$ $=2.54 \times 10^{-4}$ and $\phi_{0}=5.5$. Moreover, we have fixed the parameters at the values $g=10^{-4}, \alpha=0.99$, and $\beta=0.4$. The period doubling sequence for the phase $\phi$ when the parameter $\epsilon$ varies is shown in Fig. 6(a) while the doubling cascade for the velocity of the particle is presented in Fig. 6(b). The behavior of the Lyapunov exponent corresponding to these bifurcation diagrams is illustrated in Fig. 6(c). As we can observe the periodic orbits are characterized by negative val-
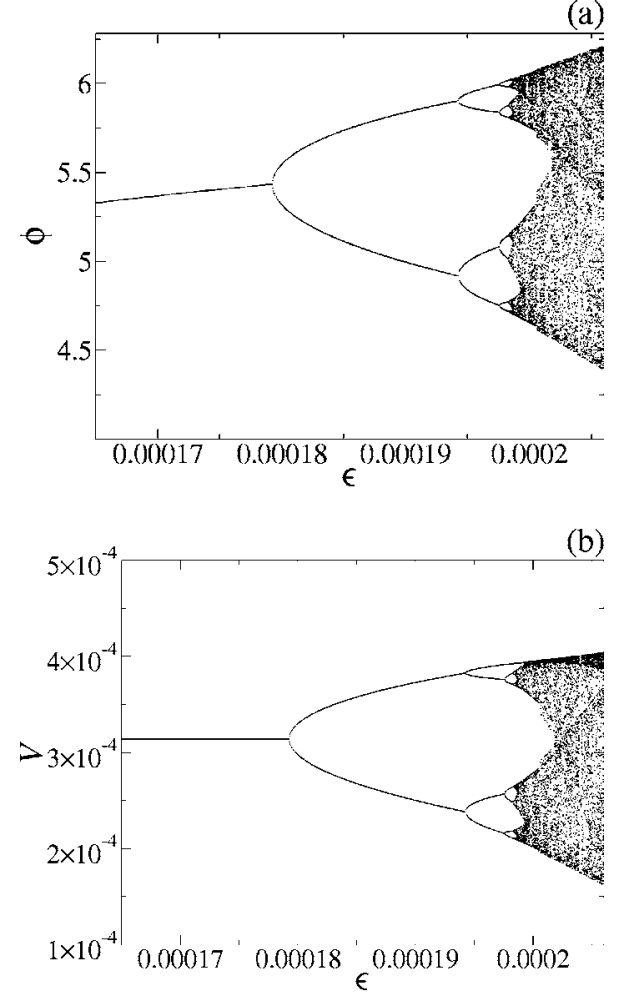

(c)

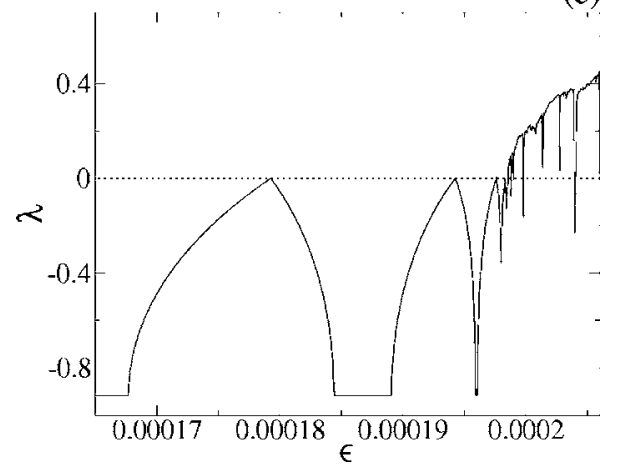

FIG. 6. Doubling cascade of (a) the phase and (b) the velocity of the particle when the parameter $\epsilon$ varies. (c) The Lyapunov exponent associated with the doubling period sequences (a) and (b).

ues of $\lambda$. It is also observed that the Lyapunov exponent goes to zero at the values of $\epsilon$ for which the bifurcations occur. As it was expected, chaotic behavior is characterized by positive values of the Lyapunov exponent. For the values of control parameters and initial conditions employed in the construction of Fig. 6, the period doubling sequence is limited to $\epsilon$ $\in\left[1.36 \times 10^{-4}, 2.06 \times 10^{-4}\right]$. Beyond these values of $\epsilon$ and due both to the high dissipation and the presence of a gravitational field, the particle experiences a locking thus moving together with the time varying wall. As we can observe in Fig. 6(b) the period-1 window for the velocity does not depend on $\epsilon$. The period-1 orbits observed in Figs. 6(a) and 6(b) correspond to the fixed point given by Eq. (10) with $m=1$. The results presented and discussed in Fig. 6 are clear evidence that the hybrid model, and for the specific ranges of control parameters used in that figure, is behaving mostly like a bouncer model. 

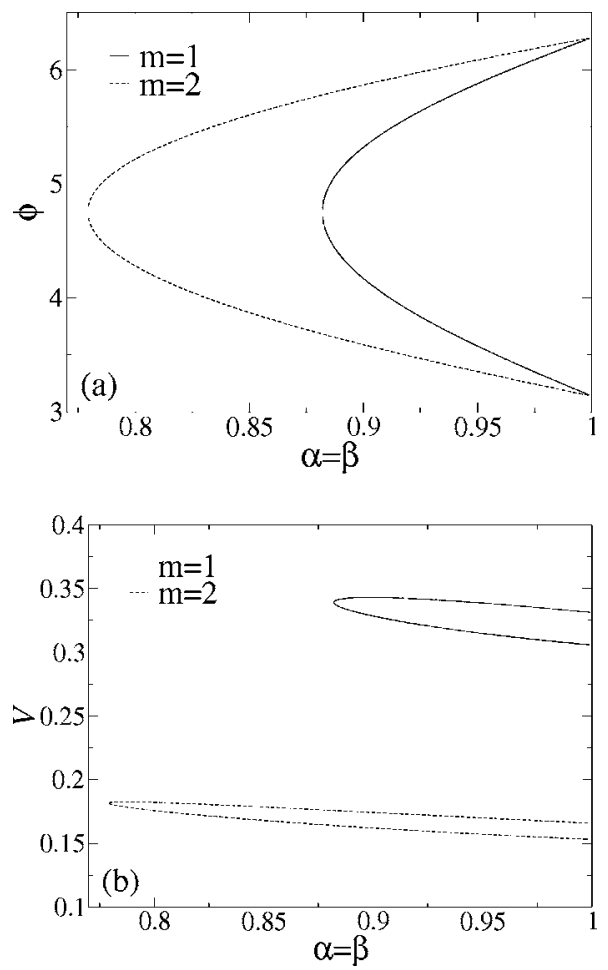

FIG. 7. Fixed points given by Eqs. (6) and (7) as a function of the damping parameters for $\alpha=\beta$. In (a) is shown the values of the phase and in (b) the values of the velocity of the fixed points. By increasing the dissipation (decreasing the values of $\alpha$ and $\beta$ ) the pair of fixed points for a given $m$ move towards each other until they become just a single fixed point and, then disappear. The control parameters used here were $\epsilon=4 \times 10^{-2}$ and $g=10^{-4}$.

\section{Annihilation of fixed points}

In this section we discuss a phenomenon directly related to the strength of the damping coefficients. It is interesting to emphasize that we can vary the damping coefficients $\alpha$ and $\beta$ while the parameters $\epsilon$ and $g$ assume constant values. An important consequence of this kind of analysis that we shall report now is the occurrence of annihilation of pairs of fixed points. As it was described in Sec. III A, from Eqs. (6) and (7) we obtain the fixed points for situation (iii). For small dissipation, these equations give us, for each value of $m$, two solutions. As an example, these solutions correspond to the saddle and the attracting fixed points, as can be seen in Fig. 1. However, by increasing the dissipation (or decreasing the values of $\alpha$ and $\beta$ ) these fixed points move towards each other and, after becoming just a single fixed point, they disappear. This annihilation phenomena is displayed in Figs. $7(\mathrm{a})$ and 7(b) where it is shown, respectively, the values of phase and velocity of the fixed points as functions of the dissipation coefficients for $\alpha=\beta$. One might also observe that for each value of $\alpha=\beta$ and for a specific value of $m$, there are two different values of fixed points. In these figures the solid lines correspond to the fixed points for $m=1$ [the curve on the right-hand side of Fig. 7(a) and the upper part of Fig. 7(b)], while the dashed lines correspond to the fixed points for $m=2$ [the curve on the left-hand side of Fig. 7(a) and the lower part of Fig. 7(b)]. The control parameters we have used were $\epsilon=4 \times 10^{-2}$ and $g=10^{-4}$. We also observe that the values of $\alpha=\beta$ for which the annihilation occurs depend on the value of $m$, too. Larger values of $m$ correspond to annihilation at high dissipation. It is also important to stress that the stability of the fixed points, which is given by the eigenvalues of the Jacobian matrix for situation (iii), depends on the values of $\alpha$ and $\beta$. Thus the fixed points could, in principle, change their classifications before being annihilated.

\section{SUMMARY AND CONCLUSIONS}

We studied the problem of a classical particle suffering inelastic collisions in the hybrid Fermi-Ulam bouncer model. The investigation was carried out in two regimes of dissipation, namely, weak and high dissipation. In the regime of weak dissipation, we characterized events of boundary crisis and in particular, obtained families of boundary crisis for different saddle points. Each family represents a practical application of an effective damping coefficient, obtained as a combination of two different $\alpha$ and $\beta$ corresponding to the dissipations on the fixed and moving wall, respectively. Such an effective damping coefficient might have applicability in experiments when dissipation cannot be neglected in practice. In the limit of high dissipation, we obtained doubling cascades as function of the two control parameters $\epsilon$ and $g$. Although we had considered the full model (without simplifications), our results indeed reinforce the previous results obtained in the literature, as can be seen, for example, in Refs. 7 and 13. Finally we also discussed the occurrences of annihilation of pairs of fixed points which were obtained by raising the strength of the dissipation.

\section{ACKNOWLEDGMENTS}

D.G.L. acknowledges the Brazilian agency CNPq for financial support. E.D.L. gratefully acknowledges CNPq and FAPESP, Brazilian agencies. We thank Diego Fregolente Mendes de Oliveira for a careful reading of our manuscript.

${ }^{1}$ A. J. Lichtenberg and M. A. Lieberman, Regular and Chaotic Dynamics (Springer Verlag, New York, 1992), Vol. 38.

${ }^{2} \mathrm{M}$. Tabor, Chaos and Integrability in Nonlinear Dynamics: An Introduction (Wiley, New York, 1989).

${ }^{3}$ E. D. Leonel and P. V. E. McClintock, J. Phys. A 38, 823 (2005).

${ }^{4}$ E. Fermi, Phys. Rev. 75, 1169 (1949).

${ }^{5}$ M. A. Lieberman and A. J. Lichtenberg, Phys. Rev. A 5, 1852 (1972).

${ }^{6}$ L. D. Pustilnikov, Trudy Moskow Mat. Obshch. 34, 1 (1977).

${ }^{7}$ R. M. Everson, Physica D 19, 355 (1986).

${ }^{8}$ M. A. Naylor, P. Sánchez, and M. R. Swift, Phys. Rev. E 66, 057201 (2002).

${ }^{9}$ P. J. Holmes, J. Sound Vib. 84, 173 (1982).

${ }^{10}$ J. M. Luck and A. Mehta, Phys. Rev. E 48, 3988 (1993).

${ }^{11}$ A. Mehta and J. M. Luck, Phys. Rev. Lett. 65, 393 (1990).

${ }^{12}$ E. D. Leonel and P. V. E. McClintock, J. Phys. A 38, L425 (2005).

${ }^{13}$ G. A. Luna-Acosta, Phys. Rev. A 42, 7155 (1990).

${ }^{14}$ E. D. Leonel and P. V. E. McClintock, Phys. Rev. E 73, 066223 (2006); J. Phys. A 39, 11399 (2006).

${ }^{15}$ D. G. Ladeira and J. K. L. da Silva, Phys. Rev. E 73, 026201 (2006).

${ }^{16}$ J. K. L. da Silva, D. G. Ladeira, E. D. Leonel, P. V. E. McClintock, and S O. Kamphorst, Braz. J. Phys. 36, 700 (2006).

${ }^{17}$ C. Grebogi, E. Ott, and J. A. Yorke, Phys. Rev. Lett. 48, 1507 (1982).

${ }^{18}$ C. Grebogi, E. Ott, and J. A. Yorke, Physica D 7, 181 (1983).

${ }^{19}$ E. D. Leonel and R. E. de Carvalho, Phys. Lett. A (to be published).

${ }^{20}$ J. P. Eckmann and D. Ruelle, Rev. Mod. Phys. 57, 617 (1985). 